\title{
a-2-Macroglobulin in Saliva as A Glycemic Control Marker in Type 2 Diabetes Mellitus Patients
}

\author{
F.M.khalil, M.A.El-Assal and A.S.Mohamed \\ Internal Medicine Dept., Faculty of Medicine, Benha Univ., Benha, Egypt \\ Email:dr_ahmed@yahoo.com.
}

\begin{abstract}
During past three decade, HbA1c has been utilized as a biomarker for assessing glycemic control and there is a need to give effortless, basic and acceptable new substitute screening strategies for DM so we intended to assess $\alpha$-2Macroglobulin in Saliva as marker for glycemic control in sort 2 diabetic patients. This cross sectional examination included all out number of 90 subjects that were ordered into 3 gatherings bunch 1with sort 2 DM with HbA1c levels more than or equivalent to $7 \%$ gathering 2 with sort $2 \mathrm{DM}$ with HbA1c levels under $7 \%$ gathering 3 healthy people as a benchmark group with fasting plasma glucose under $100 \mathrm{mg} / \mathrm{dl}, 2 \mathrm{~h}$ plasma glucose under $140 \mathrm{mg} / \mathrm{dl}$, and $\mathrm{HbA} 1 \mathrm{c}$ under $5.7 \%$.all the included cases were exposed to estimation of salivation - 2-Macroglobulin.there was high factually huge distinction between the three groupsaccording to level of salivary $\alpha 2$ macroglobulinThere was measurably critical moderate positive relationship between's $\alpha$-2-Macroglobulin with every one of FBG, 2Hr PPBG and HBA1C. With the upsides of quick, available, financially savvy, and noninvasive technique, salivary A2MG is a promising organic marker for glycemic control in patients with type 2 DM.
\end{abstract}

Keywords: Macroglobulin, Saliva, Mellitus.

\section{Introduction}

Diabetes mellitus is the third driving reason for death and because of restricted admittance to the quality medical services, the diabetic patients are more inclined to create inconveniences. Screening requires estimation of glucose levels. The best boundary is glycosylated hemoglobin (HbA1c), which gives a review of a person's glycemic control of past 4 months. Notwithstanding, this strategy is intrusive and requires blood test. The noninvasive strategy for assessment of diabetic biomarker in salivary examples is required [1].

Glycemic control is basic to deal with the illness and evade its entanglements. Fasting, $2 \mathrm{~h}$ postprandial, and glycosylated hemoglobin (HbA1c) are viewed as the strategies for assessment if there is acceptable glycemic control or not [2].

Also, alarm from sharp items (needle) can deter a few patients from checking their blood glucose levels in a customary way. It has been archived that $20.5 \%$ of patients who had needle tension stay away from all clinical treatment [3].

The noninvasive strategy for assessment of diabetic biomarker in salivary examples is required. Salivation is a wellspring of the alpha-2-macroglobulin (A2MG) other than blood. In sort 1 and type 2 diabetes mellitus (DM), the A2MG levels are expanded in blood [4].

A2MG combination is improved in diabetic patients. A2MG is delivered by liver and goes about as a plasma antiproteinase. The high serum A2MG diminishes the bioavailability of insulin, prompting weakness of blood su9gar control $[5,6]$.

The point of the current investigation was to assess $\alpha$-2-Macroglobulin in Saliva as marker for glycemic control in sort 2 diabetic patients.

\section{Patient and method}

This is a cross sectional observational examination that was led to assess $\alpha$-2-Macroglobulin in Saliva as marker for glycemic control in sort 2 diabetic patients.

This examination included all out number of 90 subjects that were ordered into 3 gatherings (utilizing the last models of American Diabetes Association):

- Group 1:

Included 40 patients with type $2 \mathrm{DM}$ with $\mathrm{HbA} 1 \mathrm{c}$ levels more than or equivalent to $7 \%$ (lacking glycemic control).

- Group 2:

Included 40 patients with type $2 \mathrm{DM}$ with $\mathrm{HbA} 1 \mathrm{c}$ levels under 7\% (sufficient glycemic control).

- Group 3:

Included 10 solid people as a benchmark group with fasting plasma glucose under $100 \mathrm{mg} / \mathrm{dl}, 2 \mathrm{~h}$ plasma glucose under $140 \mathrm{mg} / \mathrm{dl}$, and $\mathrm{HbA} 1 \mathrm{c}$ under $5.7 \%$.

\section{Incorporation rules}

- Age somewhere in the range of 30 and 60 years.

- T2DM patients as indicated by the last rules of American Diabetes Association.

Rejection rules

- Associated diabetic complexities (diabetic ketoacidosis, hyperosmolar non-ketotic unconsciousness)

- Rheumatic infections

- Nephrotic condition.

- Auto resistant sickness

- Associated intense incendiary conditions in the mouth.

- Pregnant females

- Association of other neurological illness

All patients were exposed to the accompanying:

1. Full history taking including 
- Demographic information : age, sex, unique propensities.

- Medical history :

- Associated persistent infections.

- State of ebb and flow illness od DM (beginning, course and term, intricacy and sort of treatment).

- Family history and medication history.

\section{Clinical assessment}

A. General assessment

- Vital signs (Blood pressure, beat, respiratory rate, temperature).

\section{B. Estimating of BMI}

C. Laboratory estimations:

1. Fasting blood glucose.

2. Post-brandial blood glucose.

3. Glycosylated hemoglobin (HbA1c).

4. Lipid profile.

5. Urinary egg whites/creatinine proportion.

Evaluation of salivary $\alpha$-2-macroglobulin was performed by utilizing human $\alpha 2$ macroglobulin ( $\alpha 2$ MG) ELISA Kit (Shanghai Crystal Day Biotech Co. Restricted) (Cat.No : E1097Hu).

\section{Results}

The mean age of the benchmark group was $42.17 \pm$ 5.91 years, $42.93 \pm 5.91$ years in the satisfactory glycemic control T2DM gathering and $44.93 \pm 6.81$ years in the insufficient glycemic control T2DM bunch with no critical distinction between the three gatherings $(\mathrm{p}=0.285)$. Concerning sex circulation, there were $(60 \%$, 62.5 and $67.5 \%$ guys in the control, satisfactory glycemic control T2DM and lacking glycemic control T2DM bunches individually) while there were $(40 \%, 37.5 \%$ and $32.5 \%$ females in the control, sufficient glycemic control T2DM and deficient glycemic control T2DM bunches separately) with no critical distinction in the sexual orientation appropriation of the cases remembered for the examination $(\mathrm{p}=0.545)$.

there is high measurably huge distinction in the mean BMI between the three gatherings in the investigation. The BMI was essentially higher in the uncontrolled gathering as contrasted and both the controlled diabetic gathering and wellbeing gathering. Be that as it may, the SBP was fundamentally higher in the two gatherings with T2DM either with satisfactory and lacking gatherings as contrasted and the control $(\mathrm{P}<0.001)$. The
DBP was essentially higher in the lacking glycemic control bunch as contrasted and the control $(\mathrm{p}=0.048)$.

The degree of FBG, $2 \mathrm{Hr}$ PPBG and HbA1c (\%) were measurably fundamentally higher in the Inadequate glycemic control bunch as contrasted and the control and satisfactory diabetic benchmark group. Additionally, measurably fundamentally higher in the sufficient glycemic control bunch as contrasted and the benchmark group.

All the considered clinical boundaries uncovered huge distinction between the read bunches aside from complete cholesterol and LDL

There was no critical contrast between the three gatherings with respect to the various boundaries of $\mathrm{CBC}$ including $\mathrm{Hb}$, platelets and $\mathrm{WBCs}$

the degree of Urinary Albumin-creatinine proportion was factually altogether higher in the Inadequate glycemic control bunch as contrasted and the control and satisfactory diabetic benchmark group.

the mean degree of salivary $\alpha 2$ macroglobulin in the benchmark group is $173.40 \pm 58.76 \mathrm{ng} / \mathrm{ml}$, in the satisfactory glycemic control gathering, the level was $337.90 \pm 86.95$ while in the lacking glycemic control gathering, and the level was $998.81 \pm 203.04 \mathrm{ng} / \mathrm{ml}$. there was high factually critical distinction between the three gatherings.

ECG changes were recognized in 3 cases $(7.5 \%)$ with sufficient glycemic control and in 5 cases $(12.5 \%)$ with lacking glycemic control while no cases uncovered ECG in the benchmark group. There was no measurably critical distinction between the three gatherings with respect to ECG changes.

In both the satisfactory glycemic control gathering and lacking glycemic control, there was measurably critical moderate positive connection between's $\alpha-2$ Macroglobulin with every one of FBG, 2Hr PPBG, HBA1C and BMI.

The best cutoff purpose of Salivary $\alpha 2$ macroglobulin to separate between the benchmark group and satisfactory glycemic control bunch was $226.5 \mathrm{ng} / \mathrm{ml}$ with $92.5 \%$ affectability, 80 explicitness, 94.5\% PPV, $78 \%$ NPV and $86.4 \%$ exactness.

The best cutoff purpose of Salivary $\alpha 2$ macroglobulin to separate between the satisfactory glycemic control gathering and insufficient glycemic control bunch was $521.3 \mathrm{ng} / \mathrm{ml}$ with $95.5 \%$ affectability, 98 particularity, 96.4\% PPV, 98\% NPV and $97.6 \%$ accuracy. 


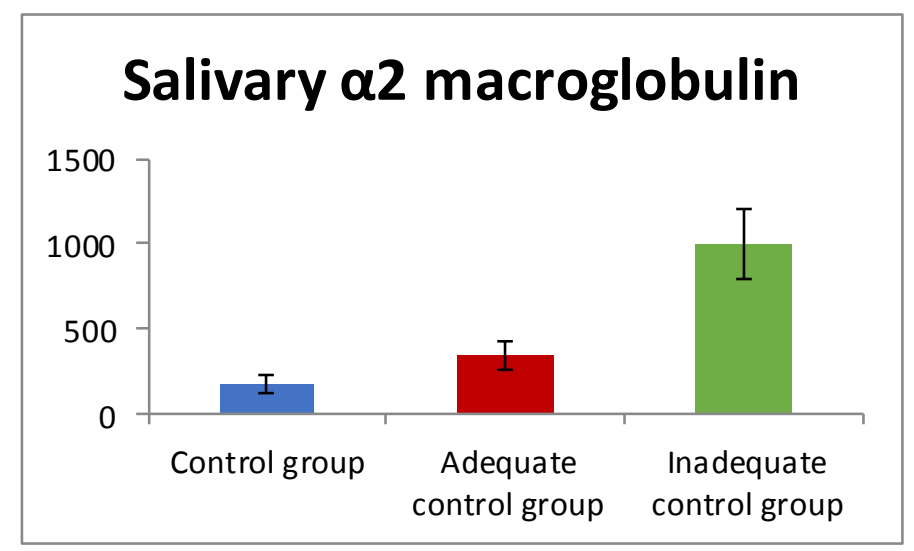

Fig (1) Level of Salivary $\alpha 2$ macroglobulin $n$ the three groups.

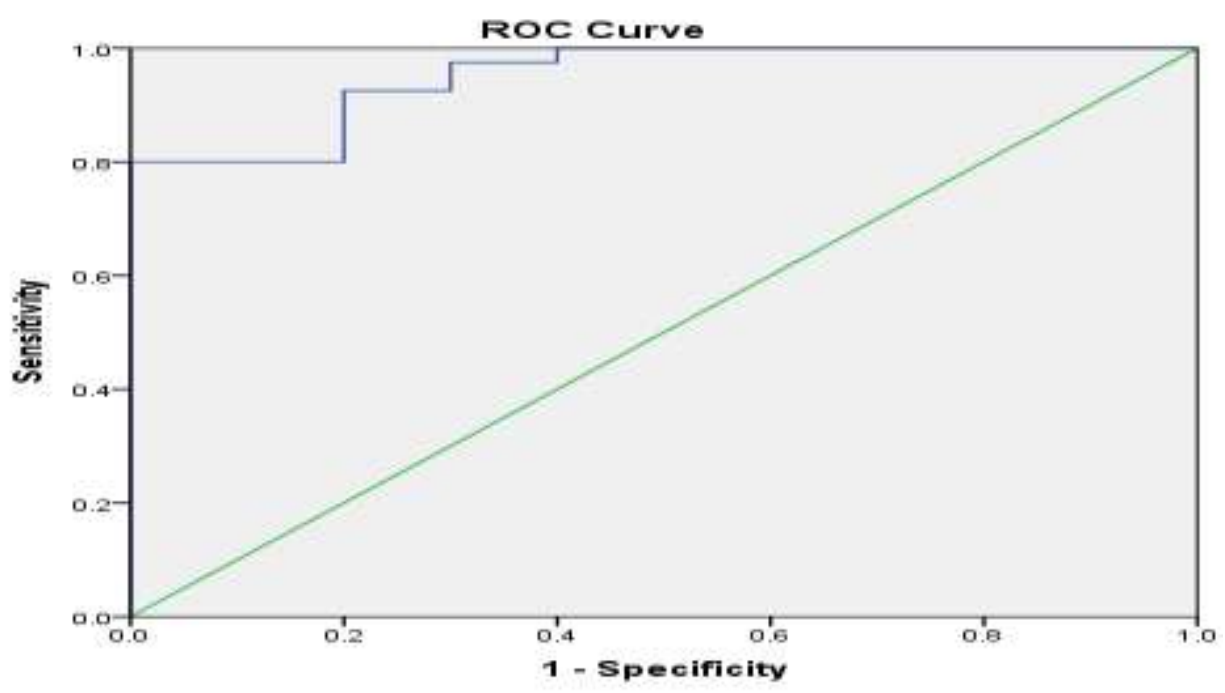

Fig (2) Roc curve of Salivary $\alpha 2$ macroglobulin to differentiate between control and adequate.

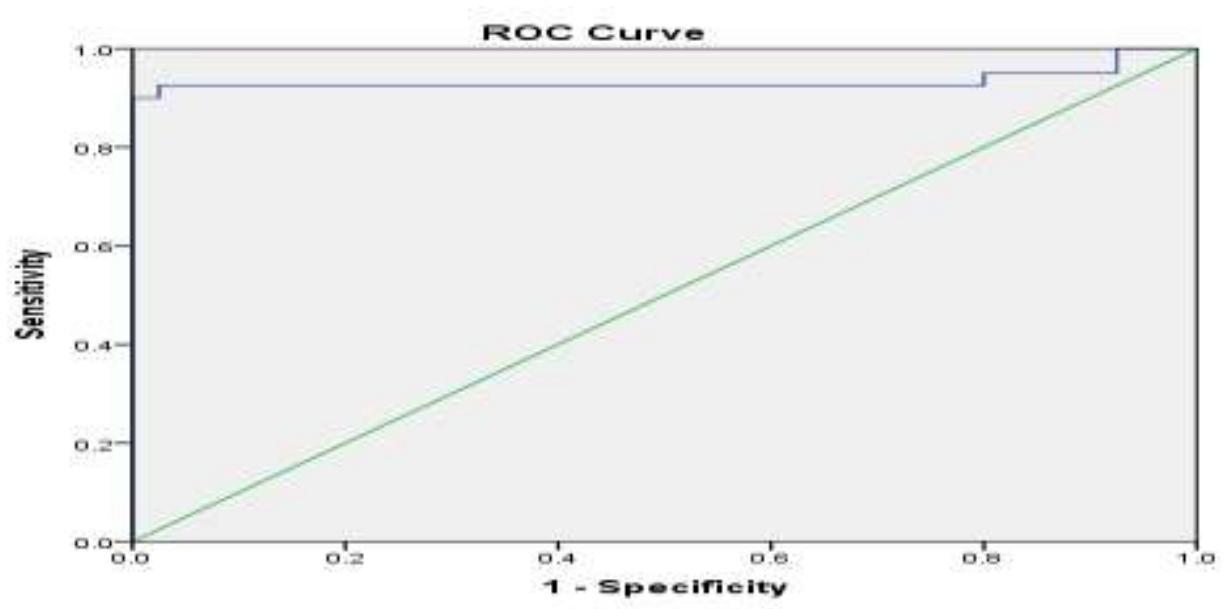

Fig (3) Roc curve of Salivary $\alpha 2$ macroglobulin to differentiate between adequate glycemic control and inadequate glycemic control groups.

\section{Discussion}

During recent decade, $\mathrm{HbA} 1 \mathrm{c}$ has been utilized as a biomarker for assessing glycemic control in sort 1 and type 2 diabetes [7,8]. The acquiring of a blood test for $\mathrm{HbA1c}$ is considered as an obtrusive methodology and is related with mental and actual affront to the patients. 
Likewise, there are numerous components which can influence HBA1c levels including race/nationality, age, hemoglobinopathies, uraemia and different elements [9].

There is a need to give easy, straightforward and agreeable new substitute screening techniques for DM recognition and order utilization of different sorts of organic examples to quantify glycemic control in sort 2 DM [10].

In this investigation, the mean degree of salivary $\alpha 2$ macroglobulin in the benchmark group is $173.40 \pm 58.76$ $\mathrm{ng} / \mathrm{ml}$, in the sufficient glycemic control gathering, the level was $337.90 \pm 86.95$ while in the insufficient glycemic control gathering, and the level was 998.81 $\pm 203.04 \mathrm{ng} / \mathrm{ml}$. there was high factually huge distinction between the three gatherings.

This came as per Nsr-Allah and his associates who announced that there were additionally high factually critical expansions in salivary A2MG levels in poor glycemic control bunch than controlled diabetic gathering and solid gathering $(\mathrm{P}<0.001)[11]$.

Our outcomes are in concurrence with the aftereffects of Chung et al., who utilized protein connected immunosorbent test for location of salivary A2MG and found that there were huge expansions in HbA1c and salivary A2MG levels in diabetic patients when 3 months of stable development to demonstrate that salivary A2MG may be utilized as a screening and observing technique for diabetes [12].

Our outcomes coordinated the aftereffects of an investigation which utilized quantative spiral immunodiffusion for discovery of serum A2MG and found that $\mathrm{A} 2 \mathrm{MG}$ was fundamentally higher in diabetic patients than in ordinary populaces and found that there was a relationship between significant level of this protein and diabetic retinopathy, which may give extra data about connection between elevated level of A2MG and retinal difficulty [12].

In addition, our information are in arrangement with another examination which utilized mass spectrometer for identifying $\mathrm{A} 2 \mathrm{MG}$ and found that $\mathrm{A} 2 \mathrm{MG}$ levels were related with glycemic control and furthermore found a connection somewhere in the range of $\mathrm{A} 2 \mathrm{MG}$ and HbA1c in 43 diabetic patients without weakness or nephropathy, since frailty may cause lower HbA1c and proteinuria may cause enormous loss of low-atomic weight proteins, bringing about height of high-subatomic weight proteins, for example, A2MG. These discoveries reinforce the theory that recognizing salivary A2MG level might be a powerful technique for checking diabetes control [13].

Another examination selected 20 members with either type 2 diabetes or prediabetes, and found that salivary proteins including A2MG demonstrated a relative expansion in plenitude with illness movement of prediabetes to the diabetic state and could be possible biomarkers for prediabetes screening [14].

Caseiro et al. have indicated that the salivary A2MG profile features the significance of the inborn insusceptible framework in the pathogenesis of type 1 diabetes and related complexities. A higher salivary
A2MG level was just found in the gathering of type 1 diabetes patients convoluted with retinopathy and nephropathy as opposed to in non-complicated type 1 diabetes patients [15].

In this examination, in both the sufficient glycemic control gathering and deficient glycemic control, there was factually huge moderate positive relationship between's $\alpha$-2-Macroglobulin with every one of FBG, $2 \mathrm{Hr}$ PPBG and HBA1C.

This came as per Nsr-Allah et al. who detailed that salivary A2MG was emphatically positivily associated with fasting blood glucose and HbAlc levels in patients with type 2 DM [11].

This comes in concurrence with the investigation of Chung et al. which proclaimed a solid positive connection among's HbA1c and both blood and salivary A2MG in patients with type 2 DM [12].

Rastogi et al. discovered connection between's spit levels of A2MG and HbA1c $(r=0.994$ and $\mathrm{P}=0.001)$ in DM2. HbA1c bunches profoundly associated with A2MG. Also, Pearson relationship coefficient was determined for $\mathrm{HbA} 1 \mathrm{c}$ and $\mathrm{A} 2 \mathrm{MG}$. It demonstrated that there was acceptable straight relationship somewhere in the range of's HbA1c and A2MG $(r=0.977, \mathrm{P}<0.001)$ [16].

Our outcomes are in accordance with those of Rao et al., which recognized higher groupings of salivary and blood A2MG in prediabetic patients contrasted and solid benchmark group, showing that there is a solid relationship between glycemic control and salivary degrees of A2MG [14].

our outcomes came in concurrence with the consequences of Aitken et al., who found a positive relationship between's salivary A2MG level and HbA1c rate $(r=0.7748$ and $\mathrm{P}<0.001)$ in patients with type $2 \mathrm{DM}$, which is roughly equivalent to our outcomes $(r=0.778$ and $\mathrm{P}<0.001$ ) [6].

Our outcomes likewise concurred with Nsr-Allah et al. who announced that salivary A2MG shows a solid positive relationship with BMI and term of diabetes in sort 2 DM [11].

Ahmad et al. discovered that in patients with type 2 diabetes, the plasma A2MG level shows an immediate positive connection with the span of diabetes and various degrees of microalbuminuria. In any case, Ahmad et al. noticed no huge connection between A2MG level with either FPG or HbA1c.

In this investigation, the best cutoff purpose of Salivary $\alpha 2$ macroglobulin to separate between the benchmark group and satisfactory glycemic control bunch was $226.5 \mathrm{ng} / \mathrm{ml}$ with $92.5 \%$ affectability, 80 particularity, $94.5 \%$ PPV, 78\% NPV and $86.4 \%$ exactness. The best cutoff purpose of Salivary $\alpha 2$ macroglobulin to separate between the sufficient glycemic control gathering and insufficient glycemic control bunch was $521.3 \mathrm{ng} / \mathrm{ml}$ with $95.5 \%$ affectability, 98 explicitness, 96.4\% PPV, 98\% NPV and $97.6 \%$ exactness.

Nsr-Allah et al. demonstrated that as per the outcomes got from ROC bend, corresponding to HBA1c 
as the highest quality level for analysis of glycemic control, ROC bend was developed at the most separating cutoff esteem $(645 \mathrm{ng} / \mathrm{ml})$ with critical territory under bend $(\mathrm{AUC}=0.92$, affectability of $91.7 \%$, particularity $90 \%$ and $\mathrm{P}<0.001)$ which was ideal to anticipate uncontrolled DM2 patients (HBA1c $\geq 7 \%$ ) showing that A2MG could be utilized as an indicative strategy for recognition of insufficient glycemic control [11].

Aitken et al. demonstrated that the region under ROC bend showed a positive segregation edge of A2MG (AUC $=0.903$, 95\% certainty stretch, $\mathrm{P}<0.001$ ), and furthermore the ideal cutoff esteem for forecast of poor glycemic control was $840 \mathrm{ng} / \mathrm{ml}$ (affectability of $81.9 \%$ and particularity of $89.6 \%$ ) for patients of uncontrolled kind 2 DM [6].

The diverse cutoff focuses announced in various investigations could be disclosed because of various standards of the included cases and various packs utilized that could uncover contrasts in their affectability.

Periodontal status is likewise related with spit levels of A2MG; indeed one examination has depicted that A2MG levels in crevicular liquid are fundamentally higher in patients with forceful periodontitis contrasted with those with persistent periodontitis [17].

For this solid affiliation, further examinations are needed for cautious evaluation of periodontal status by an oral clinical and integral investigations such as radiography.

\section{References}

[1] C.Z.Zhang, X.Q.Cheng. J.Y.Li, P.Zhang. Saliva in the diagnosis of diseases. International journal of oral science, Vol.8(3), PP.133-137,2016.

[2] S.Malkani, J.P.Mordes. Implications of using hemoglobin A1C for diagnosing diabetes mellitus. The American journal of medicine, Vol.1124(5), PP.395-401,2011.

[3] Naing, J.W.Mak. Salivary glucose in monitoring glycaemia in patients with type 1 diabetes mellitus: a systematic review. Journal of Diabetes \& Metabolic Disorders, Vol. 16(1), PP. 2,2011.

[4] I.Nakamoto, K.Morimoto, T.Takeshita. Correlation between saliva glycated and blood glycated proteins. Environmental health and preventive medicine, Vol. 8(3), PP. 95-99 .,2003.

[5] S.Sathyapriya, G.Bharani, M.Nagalingam. Potential of salivary protein as a biomarker in prognosis of diabetes mellitus. J Pharm Res, Vol. 4(7), PP. 22282229,2011.

[6] J.P.Aitken, C.Ortiz, I.Morales-Bozo. $\alpha-2-$ macroglobulin in saliva is associated with glycemic control in patients with type 2 diabetes mellitus. Disease markers .,2015.

[7] K.T.Khaw, N. Wareham, Glycated hemoglobin as a marker of cardiovascular risk. Current opinion in lipidology, Vol.17(6), PP. 637-643,2006.

[8] H.K.Holmer, L.A.Ogden, B.U.Burda. Quality of clinical practice guidelines for glycemic control in type 2 diabetes mellitus. PloS one, Vol. 8(4), PP. e58625,2013.

[9] E.Wheeler, A.Leong, C.T.Liu, , M.F.Hivert. Impact of common genetic determinants of Hemoglobin A1c on type 2 diabetes risk and diagnosis in ancestrally diverse populations: A transethnic genome-wide meta-analysis. PLoS medicine, Vol. 14(9), PP. e1002383,2017.

[10] S.Gupta, M.T.Nayak, J.Sunitha. Correlation of salivary glucose level with blood glucose level in diabetes mellitus. Journal of oral and maxillofacial pathology: JOMFP, Vol.21(3), PP. 334,2017.

[11] A.A.-E.M.Nsr-Allah, S.El-Osh, A.M.Ahmed. Salivary $\alpha 2$-macroglobulin as a marker for glycemic control in patients with type 2 diabetes mellitus. The Egyptian Journal of Internal Medicine, Vol.31(2), PP. 155, 2019.

[12] T.J.Chung, K.Y.Hsu, J.H.Chen. Association of salivary alpha 2-macroglobulin levels and clinical characteristics in type 2 diabetes. Journal of diabetes investigation, Vol. 7(2), PP.190-196, 2016.

[13] T.Takada, Y.Kodera, M.Matsubara. Serum monomeric a2-macroglobulin as a clinical biomarker in diabetes. Atherosclerosis, Vol.228(1), PP. 270-276,2013.

[14] P.V.Rao, A.P.Reddy, X.Lu. Proteomic identification of salivary biomarkers of type-2 diabetes. Journal of proteome research, Vol.8(1), PP. 239-245,2009.

[15] A.Caseiro, R.Ferreira, A.Padrao. Salivary proteome and peptidome profiling in type 1 diabetes mellitus using a quantitative approach. Journal of proteome research, Vol.12(4), PP. 1700-1709,2013.

[16] V.Rastogi, P.Kalra, M.V.Gowda. Relationship between Salivary Alpha-2 Macroglobulin and HbA1c among Patients with Type-2 Diabetes Mellitus: A Cross-sectional Study. Indian journal of endocrinology and metabolism, Vol.23(2), pp.184,2019.

[17] A.Ertugrul, H.Sahin, A.Dikilitas. Evaluation of beta-2 microglobulin and alpha-2 macroglobulin levels in patients with different periodontal diseases. Australian dental journal, Vol. 58(2), PP. 170-175,2013. 